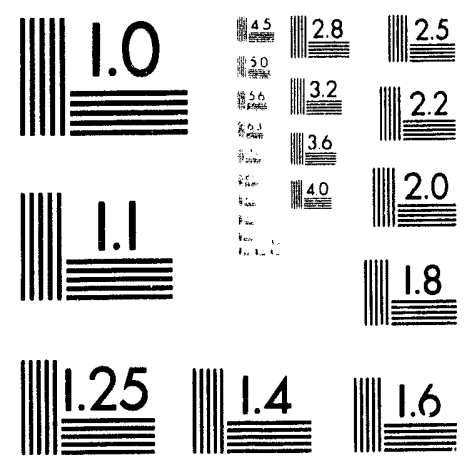



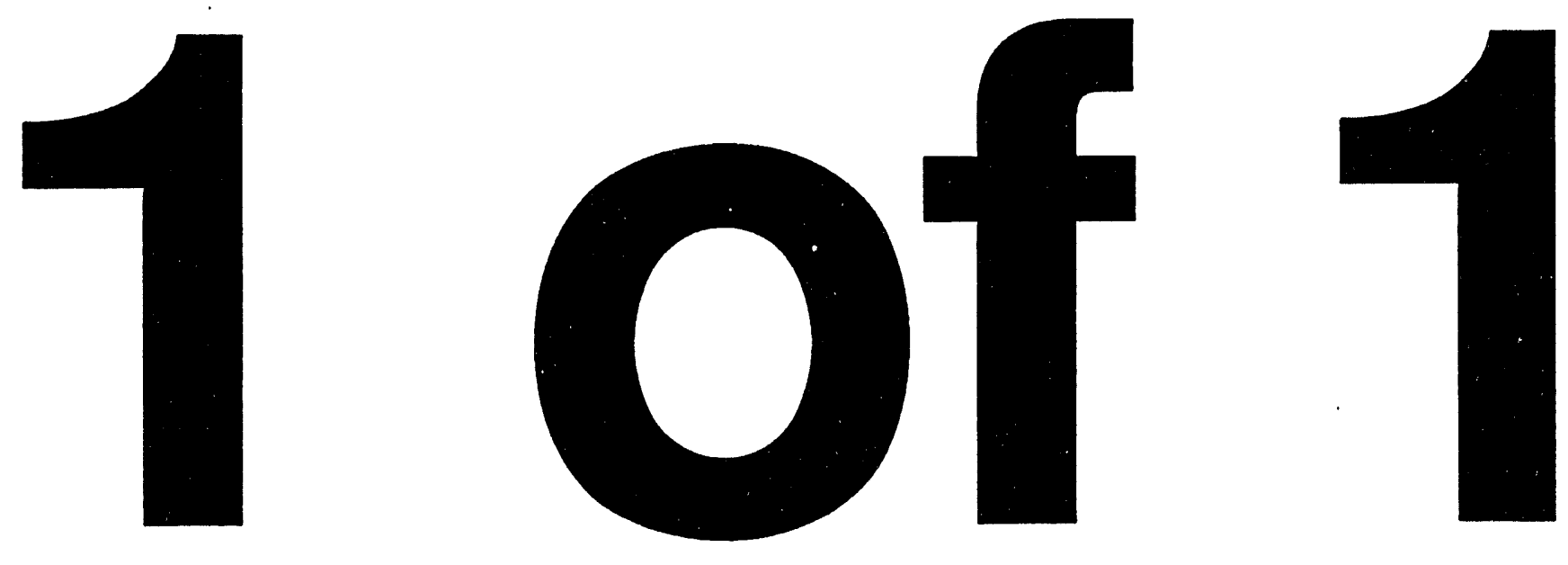


\section{DERIVATION OF A FOKKER-PLANCK EQUATION FOR BUNCHED BEAMS}

Alessandro G. Ruggiero

September 27,1993

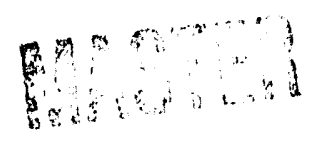




\section{Abstract *}

This report investigates the derivation of the Fokker-Planck equation which is commonly used to evaluate the evolution with time of an ensemble of particles under the effect of external rf forces, cooling and forces of stochastic nature like intrabeam scattering. The conventional approach based on the classical work by Chandrasekhar is first exposed, where the phase delay and the momentum error of the particle are used. The method is then extended to the case the distribution function is expressed in terms of the amplitude of motion instead of the original rectilinear variables. The new Fokker-Planck equation is obtained with an averaging process over the phase distribution instead of the time-averaging as it was usually performed earlier, to avoid the appearance of a singularity behavior.

The solution of the Fokker-Planck equation is chosen in the proper form which makes easier the evaluation of the beam lifetime in the presence of the separatrix of the rf buckets. Finally the numerical applications apply the Relativistic Heavy Ion Collider (RHIC).

* Work performed under the auspices of the U.S. Department of Energy 


\section{Introduction}

The Fokker-Planck equation is a useful tool to describe the evolution with time of the distribution of an ensemble of particles that move under the effect of stochastic forces, which are forces that change rapidly and discontinuously. The method for solving a stochastic differential equation, that is a Langevin equation [1], requires that some conditions are satisfied: first, the fluctuations have to take both positive and negative values with a zero average; second, the distribution of the errors is a Maxwell-Boltzmann distribution of assigned width which depends on the rate of damping and of diffusion. The major ingredient is the distribution function of the probability of finding a particle occupying a given location in the phase-space of canonically conjugated variables. Statistically this is also the distribution of an ensemble of a large number of particles in the same phase-space. Usually the variables involved assign the actual location in space and the associated velocity components. Both of these variables can take positive and negative values and have typically zero average.

An important problem, where the solution in terms of a Fokker-Planck equation is desired, is the case of a bunched beam of electrically charged particles circulating in a storage ring. The motion of the particles may be determined by the presence of external of accelerating and focussing forces, stochastic cooling and particle-particle interaction by Coulomb scattering, that is intrabeam scattering. An important issue is also the determination of the beam lifetime, or conversely the rate of beam loss due to the counteracting effects of diffusion of the motion of the particles to large amplitudes and of damping which brings the motion toward lower amplitudes. The beam loss is caused by the presence of an aperture limitation, like the separatrix of the rf buckets, since there is always a tinite chance of traversing it.

In the case of a bunched beam, the distribution changes over periods of time considerably longer than a synchrotron oscillation period. In this case, it is desired to develop a transport equation which involves only the distribution of the amplitude of the particle motion. This creates some difficulties since with the new variable the requirements above are no longer satisfied. In previous work [2-5] a Fokker-Planck equation was derived by performing a time-averaging over a synchrotron oscillation period. Unfortunately this operation introduces a singularity; in corresponcience of the separatrix, the synchrotron uscillation period diverges violating the averaying procedure itself and invalidating the evaluated behavior of the beam in proximity of the separatrix. We propose here an approach which takes the Fokker-Planck equation with the original variables and transforms it to the one for the distribution of the amplitude variable. Moreover the time average is replaced by an average over the phase distribution, avoiding the singularity. The result is an equation which has a form quite different from the one previously adopted.

In the following Section 2 we review the approach by Chandrasekhar [1] to evaluate a correct Fokker-Planck equation in terms of the original rectilinear coordinates. Section 3 discusses the analytical transformation to angle-action variables. The modified FokkerPlanck equation in terms in the new variables is derived in Section 4. The discussion of the result and the application to the Relativistic Heavy Ion Collider [7] is finally done in section 5. 


\section{Chandrasekhar's Approach to Langevin Equation}

The longitudinal component of the motion of a charged particle in a storage ring is described by a pair of canonically conjugated variables: the rf phase angle $\phi$, which measures the angular displacement around the closed reference orbit with respect to the synchronous particle, and the angular momentum $\mathrm{w}$, related to the energy error $\Delta \mathrm{E}$ by $\mathrm{w}=\Delta \mathrm{E} / \mathrm{h} \omega_{()}$where $\omega_{()}$is the angular revolution frequency, generally a function of $w$ itself. In absence of external rf forces, stochastic cooling and particle-particle interaction, the equations of motion are very simply

$\mathrm{d} \phi / \mathrm{dt}=\mathrm{aw}$

$\mathrm{d} w / \mathrm{d} t=()$

where

$a=\eta \omega_{0}^{2} h^{2} / \beta^{2} E$

with $E$ the total energy of the particle, $\beta, \gamma$ the relativistic velcicity and energy factors, $\gamma_{t}$ the value of the storage ring transition energy, $h$ the rf harmonic number which at this moment is unspecified, and $\eta=\gamma_{t}^{-2}-\gamma^{-2}$. In the following we shall assume $\gamma>\gamma_{t}$, that is the case above the transition energy when $\eta>0$ and $a>0$.

When stochastic cooling and intrabeam scattering are included, the second equation is modified as follows

$d w / d t=-\lambda w+D A(t)$

where $\lambda$ is the cooling rate and $D$ the diffusion parameter, both of which can generally include a weak and smooth dependence on time and $w$. In absence of $\mathrm{rf}$ forces, both $\lambda$ and $\mathrm{D}$ do not depend on the angle $\phi . \mathrm{A}(\mathrm{t})$ is a dimensionless, time-dependent stochastic function which changes rapidly and discontinuously.

Eq. (2.4) is called the Langevin equation. It is a stochastic differential equation subject to the restrictions on $A(t)$ stated above. The method for solving such equation is discussed in the work by Chandrasekhar [1] which we review briefly here.

In the following $\Delta t$ is a time sample short enough, during which the angular momentum $w$ does not vary appreciably. Yet $\Delta t$ is large enough to include a large number of fluctuations of the stochastic function $\mathrm{A}(\mathrm{t})$. Eq. (2.4) can then be formally integrated as follows

$\Delta \mathrm{w}=-\lambda \mathrm{w} \Delta \mathrm{t}+\mathrm{DB}(\mathrm{t})$

whire

$B(t)=\int_{t}^{t+\Delta t} A(t) d t$ 
represents the net change of $w$ which a particle may suffer on a given occasion during an interval of time $\Delta t$ around the instant $t$. This parameter is subject to statistical fluctuations described by a probability distribution function given by the following Maxwell-Boltzmann equation

$\psi(B[\Delta t])=\exp \left(-|B(\Delta t)|^{2} / 4 q \Delta t\right) /(4 \pi q \Delta t)^{1 / 2}$

where $q$ is a parameter which defines the width of the equilibrium distribution and is related directly to the cooling rate $\lambda$ and the diffusion parameter $D$. It is to be noticed that $\mathrm{B}(\Delta \mathrm{t})$ can take positive as well as negative values, that the average value expected is zero and that the distribution of the fluctuations has an rms width given by $(2 \mathrm{q} \Delta \mathrm{t})^{1 / 2}$, that is the larger the sample period $\Delta t$ the larger is the uncertainty on the magnitude of $B(\Delta t)$.

The solution of the Langevin equation (2.4) can be described by the distribution function $W(w, t)$ of the probability of finding the value $w$ at the time t. With Eqs. (2.5) and (2.7) holding, it can be proven that the distribution function satisfies the following FokkerPlanck equation

$\partial w / \partial t=\partial(\lambda w w) / \partial w+\partial^{2}(q W) / \partial w^{2}$

In the limit $t \rightarrow \infty$ this equation reduces to

$\lambda w W+\partial(q W) / \partial w=0$

which has the solution

$W(w)=\exp \left(-\lambda w^{2} / 2 q\right) /(2 \pi q / \lambda)^{1 / 2}$

The average $\bar{w}$ of the momentum distribution and the rms width $\delta^{2}$ are defined as follows

$\bar{w}=\int_{-\infty}^{+\infty} w W(w) d w$

and

$\delta^{2}=\int_{-\infty}^{+\infty} w^{2} W(w) d w$

The corresponding equations are obtained by multiplying both sides of the Fokker-Planck equation (2.8) by either $w$ or $w^{2}$ and integrating over the entire range of $w$ from $-\infty$ to $+\infty$. One finds

$\mathrm{d} \overline{\mathrm{w}} / \mathrm{d} \mathrm{t}=-\lambda \overline{\mathrm{w}}$

$d \delta^{2} / \mathrm{dt}=-2 \lambda \delta^{2}+2 \mathrm{q}$

which has the following equilibrium value in the limit $t \rightarrow \infty$

$\delta_{\infty}^{2}=\mathrm{q} / \lambda$ 
in agreement with Eq. (2.10). Eq. (2.14) should be compared with the original Eq. (2.4). It is seen that q replaces $D$. It is the proper diffusion coefficient defined as the average increase of $w^{2}$ per unit of time.

We want to emphasize that the derivation of the Fokker-Planck equation (2.8) is based on the assumption of the distribution function (2.7) of the statistical fluctuations, and applies correctly to a variable $w$ which takes both positive and negative values. In particular the average value of $w$ is zero.

It is also to be noticed that the angle coordinate $\phi$ does not enter the Fokker-Planck equation, and remains as a "spectator" variable. Indeed the action of stochastic cooling and of particle-particle interaction affects only the momentum variable w. It is, nevertheless, possible to modify the Fokker-Planck equation to include formally also the dependence on the variable $\phi$. If the distribution function is $W=W(\phi, w, t)$ the corresponding equation is

$\partial w / \partial t+a w \partial w / \partial \phi=\partial(\lambda w w) / \partial w+\partial^{2}(q w) / \partial w^{2}$

It is also possible to include an external, rf restoring force which modifies Eq.(2.4) as follows

$d w / d t=-\lambda w-b \sin \phi+D A(t)$

where, for the case of non-accelerating, stationary rf buckets,

$\mathrm{b}=\mathrm{Qe} \mathrm{V} / 2 \pi \mathrm{hA}$

with $Q$ the charge state, $A$ the mass number of the particle and $V$ the peak rf voltage. For this case the Fokker-Planck equation modifies as follows

$\partial w / \partial t+a w \partial w / \partial \phi-b \sin \phi \partial w / \partial w=\partial(\lambda w w) / \partial w+\partial^{2}(q w) / \partial w^{2}$

which is the generalization of the Liouville's theorem according to Chandrasekhar.

Eq. (2.19) is the correct representation of the evolution of a bunch of charged particles moving in a storage ring under the action of the external rf forces, stochastic cooling and intrabeam scattering. It can be expected that the cooling rate $\lambda$ and the diffusion coefficient proper $q$ have also a dependence on the azimuthal angle $\phi$, since they depend on the actual location of the test particle within the bunch.

\section{Transformation to Angle-Action Variables}

The unperturbed equations of motion in the presence of the external rf forces

$$
\begin{aligned}
& \mathrm{d} \phi / \mathrm{dt}=\mathrm{aw} \\
& \mathrm{dw} / \mathrm{d} \mathrm{t}=-b \sin \phi
\end{aligned}
$$


can be derived from the Hamiltonian

$H=a w^{2} / 2+2 b \sin ^{2} \phi / 2$

through the Hamilton's equations

$\mathrm{d} \phi / \mathrm{d} t=\partial \mathrm{H} / \partial \mathrm{w}$

$\mathrm{dw} / \mathrm{dt}=-\partial \mathrm{H} / \partial \phi$

Since the Hamiltonian does not depend explicitly on time, it is more convenient to operate a canonical transformation from the pair of variables $(\phi, w)$ to the pair $(Q, J)$ also of canonically conjugated variables, through the generating function

$S=S(\phi, J)=\int \phi d x \sqrt{(2 / a)\left[J-2 b \sin ^{2}(x / 2)\right]}$

which gives

$\mathrm{w}=\partial \mathrm{S} / \partial \phi$
$\mathrm{Q}=\partial \mathrm{S} / \partial \mathrm{J}$

The variable $\mathrm{J}$ is identified with the Hamiltonian itself, that is

$J=a w^{2} / 2+2 b \sin ^{2} \phi / 2$

and

$Q=\int \phi d x\left\{(2 a)\left[J-2 b \sin ^{2}(x / 2)\right]\right\}^{-1 / 2}$

$\mathrm{J}$ is the amplitude of the particle oscillatory motion and the variable $\mathrm{Q}$ is the time required to travel around the trajectory of amplitude J. To complete one oscillation, it takes a period $\mathrm{T}=2 \pi / \Omega$ which is a function of $\mathrm{J}$. The phase oscillation angular frequency is given by

$\Omega=\pi \Omega_{0} / 2 K(\mathrm{k})$

where

$\Omega_{1}=(\mathrm{a} \mathrm{b})^{1 / 2}$

is the frequency in the limit of small oscillations and $K(\mathrm{k})$ is the complete elliptical integral of the first kind [8] with

$k=(J / 2 b)^{1 / 2}$

The unperturbed equations of motion (3.4) and (3.5) are now replaced by

$\mathrm{d} Q / \mathrm{d} \mathrm{t}=1$ 
The variable $\mathrm{J}$ is a non-negative quantity which takes the value zero in correspondence to the center of the beam bunch and increases in value with increasing trajectory size until it reaches the value $\mathrm{J}_{\mathrm{s}}=2 \mathrm{~b}$ in correspondence of the separatrix. Beyond the separatrix the motion is unstable. At the center of the bunch $\mathrm{k}=0, K=\pi / 2$ and the angular frequency of the phase oscillations $\Omega=\Omega_{0}$. At the separatrix, $\mathrm{k}=1, K \rightarrow \infty$ and $\Omega=0$, as it is well known.

\section{Fokker-Planck Equation in the Action Variable}

It is desirable to express the Fokker-Planck equation (2.19) in terms of the new transformed variables $Q$ and $J$ through a probability distribution function $W(Q, J, t)$. Since $J$ is a non-negative quantity, and its statistical fluctuations do not satisfy the Maxwell-Boltzmann distribution (2.7), one cannot derive the corresponding Fokker-Planck equation $a b$ initio as done for the distribution $\mathrm{W}(\phi, w, t)$ in terms of the variables $\phi$ and $w$. We propose here the following approach.

The phase oscillation period $\mathrm{T}_{0}=2 \pi / \Omega_{0}$ in the center of the bunch is considerably larger than the sampling time $\Delta \mathrm{t}$ we take to measure the net change due to the statistical fluctuations. Moreover the effects due to stochastic cooling and intrabeam scattering evolve over periods of time that, though comparable to each other, are both longer than $\mathrm{T}_{0}$. Therefore it is reasonable to assume that the bunch distribution is continuously matched to the shape of the trajectories in the $(\phi, w)$-phase plane and that the distribution function depends explicitly only on $J$ and not on $Q$, that is $W=W(J, t)$. This simplifies considerably the left-hand side of Eq. (2.19) since, from Eqs. (3.3-3.5) and (3.9)

$a w \partial w / \partial \phi-b \sin \phi \partial w / \partial w=$

$=\{(d \phi / d t)(\partial J / \partial \phi)+(d w / d t)(\partial J / \partial w)\}(\partial W / \partial J)=0$

The right-hand side of Eq. (2.19) can also be readily transformed in terms of the variable $J$. The two quantities $\lambda$ and $q$ may include a dependence on $w$, but weak enough so that they can be taken in and out of the differential operator $\partial / \partial w$ without altering the result considerably. Moreover, they have a dependence on $\phi$, mostly through the actual location within the bunch. Their values have at very most a factor of 2 difference between the center of the bunch and the outer edge where particles sweep through the center twice each phase oscillation. Thus, since we are interested in the overall behavior of the distribution and not in that of a single particle, it is a good approximation to replace $\lambda$ and $q$ with about half the peak value they take at $\phi=0$. Finally the differential operator $\partial / \partial w$ can be easily transformed to the operator $\partial / \partial \mathrm{J}$

$\partial(\lambda w W) / \partial w \sim \lambda w+\lambda\left(a w^{2}\right)(\partial W / \partial J)$

$\partial^{2}(q W) / \partial w^{2} \sim q a(\partial W / \partial J)+q a\left(a w^{2}\right)\left(\partial^{2} W / \partial J^{2}\right)$

where 


$$
\left(a w^{2}\right)=2 J-4 b \sin ^{2}(\phi / 2)
$$

With the same arguments as before, the last equation is to be evaluated at the center of the hunch and reduced by a factor of 2 . In conclusion

$$
\partial W / \partial t=\partial\{J \mid \lambda W+\partial(\text { a } W) / \partial J]\} / \partial J
$$

which is, in our opinion, a more valid Fokker-Planck equation for the distribution function $\mathrm{W}(\mathrm{J}, \mathrm{l})$ given in terms of the amplitude of motion $\mathrm{J}$. Our procedure, which is hased on the averaging over phases, avoids the averaging over time, that is over synchrotron oscillation periods, that would introduce a singularity hehavior around the separatrix [2-3,5].

Eq. (4.5) involves only coherent and stochastic variation $\Delta \mathrm{J}$ with $\Delta t$ through the cooling rate $\lambda$ and the diffusion coefficient proper 4 . The nature of the physical problem being investigated does not require introducing the average variation of $\left\langle\Delta \mathrm{J}^{2}\right\rangle$ over $\Delta \mathrm{t}$.

For the case of debunched heam, in absence of rf external forces, i. e. $b=0$, we have derived the correct Fokker-Planck equation (2.16). There is no dependence of this equation with the phase angle $\phi$; thus, the transformation procedure from the angular momentum $w$ to the invariant $\mathrm{J}=\mathrm{aw}^{2} / 2$ is more rigorous and gives exactly the transformed Fokker-Planck equation (4.5).

In the limit $t \rightarrow \infty$ Eq. (4.5) exhihits the following equilibrium behavior

$\lambda W+\partial(q a W) / \partial J=0$

which has the stationary solution

$\mathrm{W}(\mathrm{J})=\exp \left(-\mathrm{J} / \mathrm{J}_{\infty}\right) / \mathrm{J}_{\infty}$

where

$\mathrm{J}_{\infty}=\mathrm{q} \mathrm{a} / \lambda$

is the median value of the distribution at the equilibrium. We can also define the average

$J=\int_{0}^{\infty} J W(J, t) d J$

The equation for $\mathrm{J}$ is obtained by multiplying both sides of $\mathrm{Eq}$. (4.5) by $\mathrm{J}$ and integrating over the range of $\mathrm{J}$ from () $10 \infty$

$\mathrm{d} J / \mathrm{dl}=-\lambda J+4 \mathrm{a}$

which exhibits the asymptotic value $\mathrm{J}=\mathrm{J}_{\infty}$ in agreement with Eqs. (4.7) and (4.8). Furthermore, reminding the relation between $J$ and $w$, it is seen that Eq. (4.10) can be identified with Eq. (2.14), and that hoth equations yield the same equilibrium value for the width of the distribution. 


\section{Integration of the Fokker-Planck Equation. Beam Lifetime.}

In principle, the Fokker-Planck equation (4.5) is to be solved by assigning an initial distribution $W(J)$ at $t=0$, when the initial number of particles is $N_{0}$, and integrating by requiring that at the separatrix the distribution is zero at all times, that is

$\mathrm{W}\left(\mathrm{J}=\mathrm{J}_{\mathrm{s}}\right)=0$

reminding that $J_{S}=2 b$ and that $b$ is given by Eq. (2.18). Integration of $W(J, t)$ over all values of $\mathrm{J}$, from () to $\mathrm{J}_{\mathrm{s}}$, gives the fraction $\mathrm{N} / \mathrm{N}_{0}$ of particles surviving at the time $t$.

We shall attempt here an analytical solution of Eq. (4.5) by representing the solution as the sum over fundamental modes, that is by choosing

$\mathrm{W}(\mathrm{J}, \mathrm{t})=\sum_{\mathrm{S}} C_{\mathrm{S}} \exp \left(-\beta_{\mathrm{S}} \mathrm{t}\right) \mathrm{U}_{\mathrm{s}}(\mathrm{J})$

where $C_{s}$ and $\beta_{s}$ are integration constants that describe the fundamental modes and the initial distribution at $t=0$. The eigenmodes are described by the function $U_{S}(J)$ which are chosen so to satisfy the boundary condition

$\mathrm{U}_{\mathrm{s}}(\mathrm{J})=0$ at $\mathrm{J}=\mathrm{J}_{\mathrm{S}}$

Inserting the series expansion Eq. (5.2) in the Fokker-Planck Eq. (4.5) gives the following equation for the eigenfunction $\mathrm{U}_{\mathrm{s}}(\mathrm{J})$

$\alpha \mathrm{Jd}^{2} \mathrm{U}_{\mathrm{s}} / \mathrm{dJ}^{2}+(\mathrm{J}+\alpha) \mathrm{d} \mathrm{U}_{\mathrm{s}} / \mathrm{d} \mathrm{J}+\left(1+\mathrm{f}_{\mathrm{s}}\right) \mathrm{U}_{\mathrm{s}}=0$

where

$\alpha=\mathrm{q} a / \lambda$

and

$\mathrm{f}_{\mathrm{s}}=\beta_{\mathrm{s}} / \lambda$

Eq. (5.4) is Kummer's equation [8] which has as independent solutions the two Kummer's functions $M$ and $U$, of which though only the first can be accepted since the second has a singularity behavior at the origin $\mathrm{J}=0$. Thus

$\mathrm{U}_{\mathrm{s}}(\mathrm{J})=M\left(-\mathrm{f}_{\mathrm{S}}, \mathrm{l}, \mathrm{J} / \alpha\right) \exp (-\mathrm{J} / \alpha)$

The Kummer's function $M$ has the s-th positive zero which in good approximation [8] is given by

$x_{s}=\pi^{2}(s-1 / 4)^{2} /\left(2+4 f_{s}\right)$

The eigenmodes are determined by imposing the boundary condition given by Eq. (5.3), that is 
$\beta_{\mathrm{s}}=\pi^{2} \mathrm{qa}(\mathrm{s}-1 / 4)^{2} /\left(4 \mathrm{~J}_{\mathrm{s}}\right)-\lambda / 2$

This is made of two terms: a contribution from the diffusion rate (a q) which is positive, and a contribution from the cooling rate $\lambda$ which is negative. As expected, the former causes a decrease of the density of the core of the distribution and the latter an increase.

The several modes which appear in Eq. (5.2) can be used to evaluate the evolution of the distribution from its initial configuration. In absence of cooling $(\lambda=0)$, after an initial period of time where the distribution widens and reaches the separatrix of the rf buckets, the overall behavior is essentially determined by the lowest eigenmode corresponding to $s=1$, since all the other modes will have in the meantime decayed very quickly. Correspondingly the beam lifetime $\tau_{b 0}$ itself can be identified with the inverse of the growth rate $\beta_{1}$, that is

$\tau_{\mathrm{b} 0}=128 \mathrm{~b} /\left(9 \pi^{2} \mathrm{qa}\right)$

This is verified with the numerical integration of the Fokker-Planck equation itself that in absence of cooling $(\lambda=0)$, can also be written as

$\partial w / \partial \tau=\partial w / \partial u+u \partial^{2} w / \partial u^{2}$

where we have introduced the reduced variables $\mathrm{u}=\mathrm{J} / \mathrm{J}_{\mathrm{S}}$ and $\tau=\mathrm{t} / \mathrm{T}_{\mathrm{S}}$ with $\mathrm{T}_{\mathrm{s}}=2 \mathrm{~b} / \mathrm{q}$ a. The condition (5.1) translates to $\mathrm{W}(1)=0$. The result of the numerical integration is shown in Figure 1 which displays the distribution function $W(u)$ versus $u$ in the range between 0 and 1 . The top curve is the initial distribution at $\tau=0$ and the one at the bottom corresponds to $\tau=1$. The other curves are at instants in between taken at $\tau=0.1$ apart. The vertical scale is in arbitrary linear units. Integration and normalization of the distribution $W(u)$ over the range $0<u<1$ at a given instant $\tau$ gives the number of particles survived at the same instant which is plotted in Figure 2 versus $\tau$ between 0 and 1 . It is seen that beam lifetime is about $0.7 \mathrm{~T}_{\mathrm{s}}$, in agreement with Eq. (5.10).

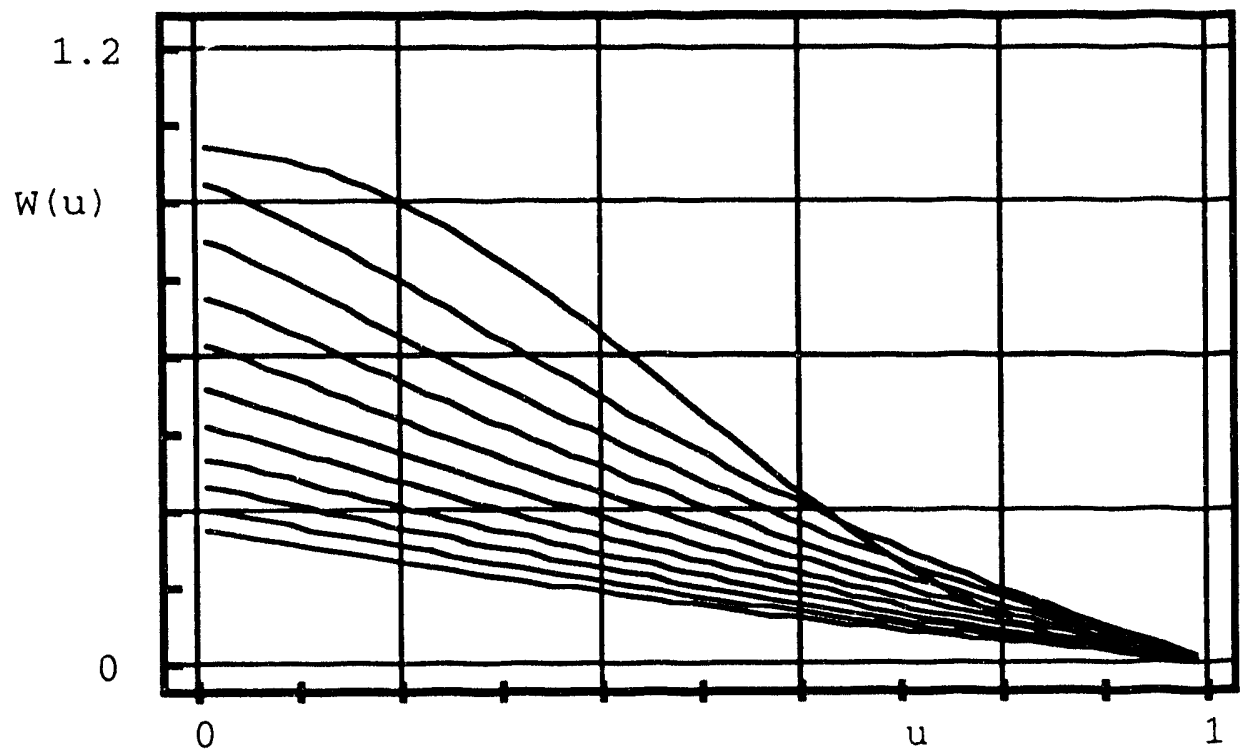

Fig. 1. Evolution of the Distribution Function W(u) vs. time in absence of Cooling 


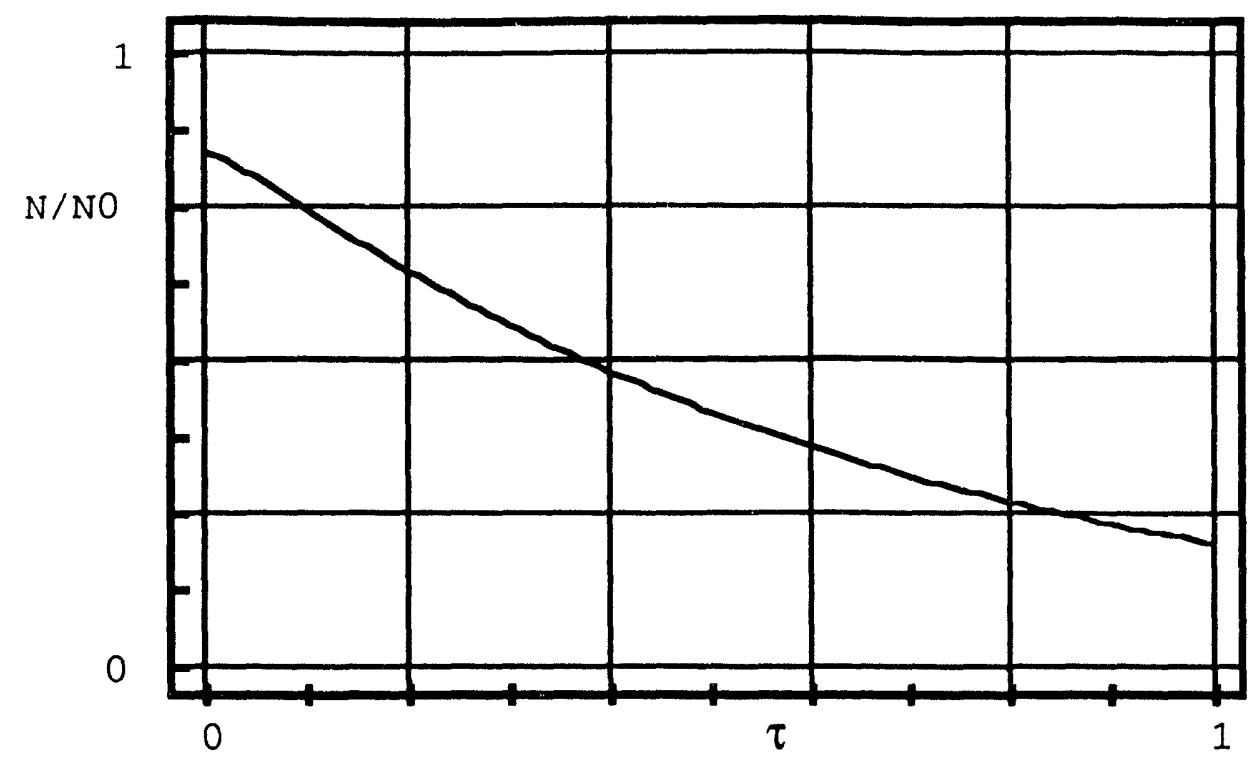

Fig. 2. Beam Survival vs. Time in absence of Cooling

If stochastic cooling is added at the rate $\lambda$, a reasonable criterion is to adjust the cooling rate so to obtain the required beam width $J_{\infty}$. From Eq. (4.8)

$\lambda=\mathrm{qa} / \mathrm{J}_{\infty}$

In this case the beam lifetime, which we shall denote $\tau_{b c}$, after the initial transition period, is determined again by the lowest mode $s=1$, which gives, in good approximation,

$\tau_{\mathrm{bc}}=\tau_{\mathrm{b} 0} /\left(1-0.36 \mathrm{~J}_{\mathrm{s}} / \mathrm{J}_{\infty}\right)$

If the cooling rate is adjusted so that $\mathrm{J}_{\infty}>0.36 \mathrm{~J}_{\mathrm{s}}$, the diffusion process will dominate resulting in a lowering and widening of the distribution and of beam particle loss at the rate given by $\tau_{b c}{ }^{-1}$. If on the contrary $J_{\infty}<0.36 J_{s}$, the cooling process will dominate, causing an increase and narrowing of the distribution at the rate $\left|\tau_{b c}\right|^{-1}$. There are also no beam losses, and the beam lifetime is infinite. This case is also verified with the numerical integration of the Fokker-Planck equation that can be written also as follows

$\partial W / \partial \tau=W+(u+\alpha) \partial W / \partial u+\alpha u \partial^{2} w / \partial u^{2}$

where $\mathrm{u}=\mathrm{J} / \mathrm{J}_{\mathrm{S}}$ as before, now $\alpha=\mathrm{J}_{\infty} / \mathrm{J}_{\mathrm{s}}$ and $\tau=\lambda \mathrm{t}$. The result of the numerical integration of Eq. (5.14) is shown in Figure 3 for $\alpha=0.1$. The lower curve is the initial distribution at $\tau=0$ and the top one corresponds to $\tau=1$. All the others in between are taken at $\tau=0.1$ apart. By numerically integrating the distribution functions it has been determined that indeed the beam intensity is constant versus time.

It is seen that the optimum from the beam lifetime point of view is obtained by letting $\beta_{1}=0$, that is by setting the cooling rate so that

$\mathrm{J}_{\infty}=0.32 \mathrm{~J}_{\mathrm{s}}=0.64 \mathrm{~b}$ 
which requires

$\lambda=1.56 \mathrm{qa} / \mathrm{b}$

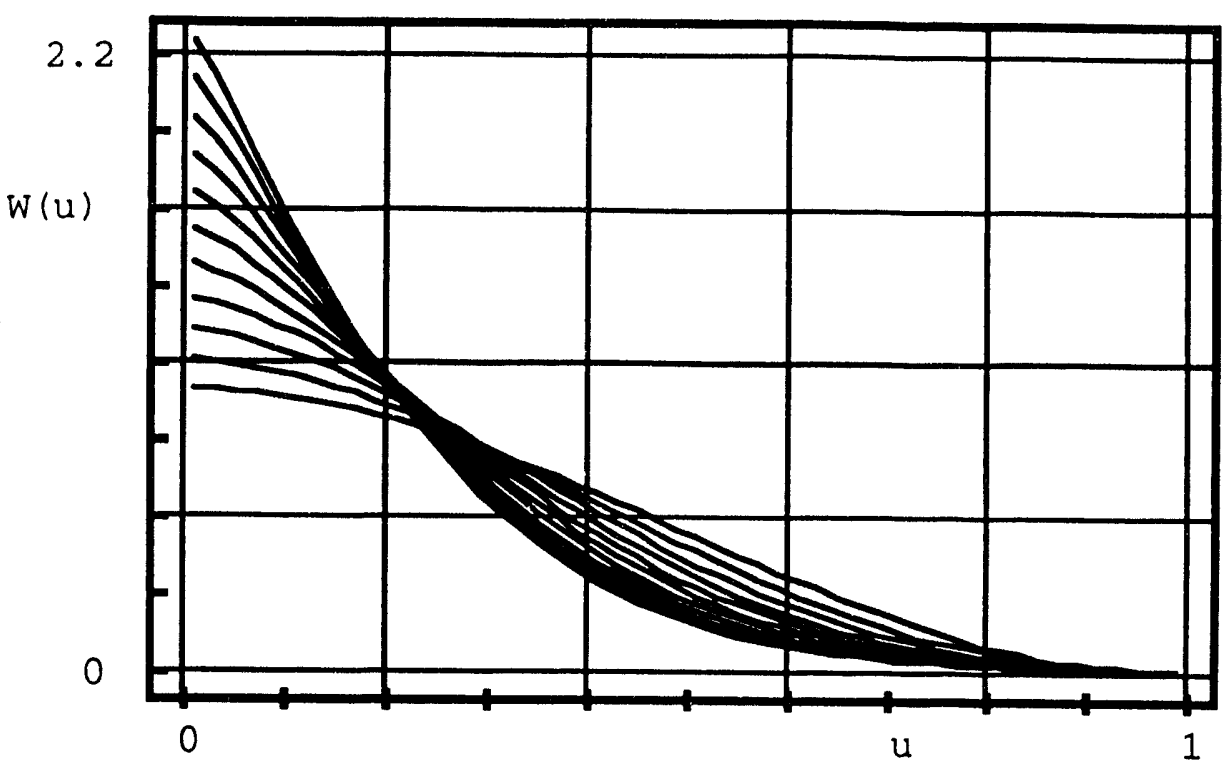

Fig. 3. Evolution of the Distribution Function W(u) vs. Time with Cooling

It is to be observed that all the major results of this section involve the constant $b$, which is given by Eq. (2.18), and that therefore depend on the peak of voltage $V$ and on the rf harmonic number $h$. The other important parameter is the kinematic constant a given by Eq. (2.3). The diffusion rate $q$ is defined through Eq.s (3.9) and (4.10) and is related to the rms increase of $w^{2}$ per unit of time, that is $2 \mathrm{q}=\mathrm{d}\left\langle\mathrm{w}^{2}\right\rangle / \mathrm{dt}$ or $2 \mathrm{~h}^{2} \omega_{0}^{2} \mathrm{q}=\mathrm{d}\left\langle\Delta \mathrm{E}^{2}\right\rangle / \mathrm{dt}$.

\section{Application to RHIC}

The performance of the Relativistic Heavy Ion Collider [7] is greatly influenced by the phenomena of Intrabeam Scattering which is the one made of a sequence of events with stochastic behavior and which gives rise to a diffusion process at the rate we have denoted before by q. This phenomena nevertheless evolves over very long periuds of time, involving several synchrotron oscillations before an appreciable effect of the beam can be noticed which is consistent with the assumption we have made in the paper. Similarly, stochastic cooling of the bunched beam can be added but also with comparatively long cooling periods. In this mode of operation, which requires relatively low electronic gain, the diffusion effects due to both Schottky and thermal noise can be neglected with respect to the stochastic effects of the particle-particle scattering [4]. Thus the all process can very well be described only by a cooling rate $\lambda$ and a diffusion rate $\mathrm{q}$ which at the limit can be taken constant or very slowly varying. The cooling rate $\lambda$ is proper of the longitudinal stochastic cooling system, and the diffusion rate $q$ is proper of the intrabeam scattering phenomena. 
The diffusion rate $\mathrm{q}$ depends on the beam betatron emittance $\varepsilon$, on the beam intensity $\mathrm{N}$, and on the bunch length and momentum spread. Thus there is to some degree also a dependence on the rf parameters, like of peak voltage $\mathrm{V}$, the of harnionic number $\mathrm{h}$ and the compaction parameter $\eta$ of the storage ring.

The reference is given by the case of a bunched beam of fully stripped ions of gold (Au). The main parameters are reported below

Mass Number, A

Charge State, Q

Rest Energy, $\mathrm{E}_{0}$

Kinetic Energy

$\beta$

$\gamma$

Transition Energy, $\gamma_{t}$

$\eta$

Circumference

Revolution Frequency, $\omega_{0} / 2 \pi$

rf Harmonic Number, $h$

rf Peak Voltage, $V$

Number of Ions / Bunch, $\mathrm{N}_{\mathrm{o}}$
197

79

$0.93113 \mathrm{GeV} / \mathrm{u}$

100

0.99996

108.4

24.7

0.001554

3833.852

78.193

m

2052

4.5
$1 \times 10^{9}$

From which $\mathrm{a}=15,650(\mathrm{eV} / \mathrm{u})^{-1} \mathrm{~s}^{-2}, \mathrm{~b}=140 \mathrm{eV} / \mathrm{u}$ and $\mathrm{J}_{\mathrm{s}}=280 \mathrm{eV} / \mathrm{u}$. The rf bucket full height is $\Delta \mathrm{p} / \mathrm{p}=4.0 \times 10^{-3}$. If the normalized betatron emittance $\varepsilon_{\mathrm{H}}=\varepsilon_{\mathrm{V}}$ is enlarged at the beginning of the storage to the full value of $60 \pi \mathrm{mm}-\mathrm{mrad}$, and the longitudinal area of the bunch has a full value of $0.3 \mathrm{eV} / \mathrm{u}-\mathrm{s}$ at the start, then the betatron emittance grows very little during a 10-hour long storage: up to about $70 \pi \mathrm{mm}$-mrad. At the same time the beam momentum spread increases at about a constant rate which corresponds to $\mathrm{q}=1 \times 10^{-7}(\mathrm{eV} / \mathrm{u})^{2} / \mathrm{s}$. The result of the computation of the growth [6], from which the value of the diffusion rate $q$ can be inferred, is displayed in Figure 4 which plots the square of the bunch momentum spread versus time in the period between 2 and 10 hours of a typica! storage.

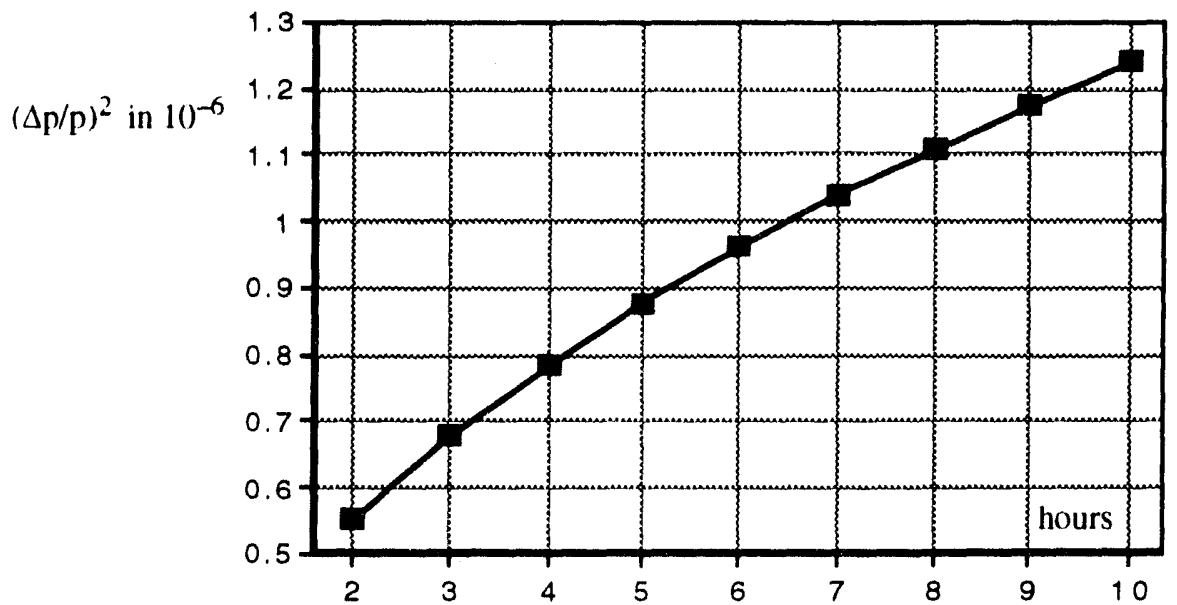

Fig. 4. The square of the Bunch Momentum Spread vs. Time during a Storage 
It is immediately derived that $\mathrm{q} a=0.0016(\mathrm{eV} / \mathrm{u}) / \mathrm{s}$. In absence of stochastic cooling $(\lambda=0)$ the beam lifetime is $\tau_{b x}=0.7 T_{s}=34$ hour. After a storage which is 10 hours long only about $75 \%$ of the beam survives.

Stochastic cooling can be added to lengthen the beam lifetime, essentially to infinite. According to Eq. (5.15) the cooling time required is $\tau_{\text {cooling }}=\lambda^{-1}=0.32 \mathrm{~T}_{\mathrm{s}}=15.6 \mathrm{hrs}$. More generally if one requires reducing the bunch dimensions to the ratio $\alpha=\mathrm{J}_{\infty} / \mathrm{J}_{\mathrm{s}}$ then the required cooling time is $\tau_{\text {cooling }}=(48.6 \mathrm{hrs}) \alpha$. For instance, the cooling time required for $\alpha=0.1$ is about $4.9 \mathrm{hrs}$.

\section{References}

[1] S. Chandrasekhar, Rev. of Mod. Phy., 15, no. 1, January 1943

[2] J. Wei, Stochastic Cooling in the RHIC. Contribution to this Workshop.

[3] J. Wei and A. G. Ruggiero, "Intrabeam Scattering and the Beam Life-Time", Informal Report BNL-45269 and AD/RHIC-81, September 1990

[4] J. Wei and A. G. Ruggiero, "Beam Life-Time with Intrabeam Scattering and Stochastic Cooling", Conference Paper BNL-45515 and AD/RHIC-90, May 1991. IEEE 1991 PAC , 91CH3().38-7, vol. 3, pages 1869-1871

[5] S. V. Ivanov, "Longitudinal Diffusion of a Proton Bunch under External Noise", Preprint IHEP 92-43 (UNK), Protvino, 1992

[6] G. Parzen, Private Communication

[7] Conceptual Design of the Relativistic Heavy Ion Collider RHIC, BNL 52195. May 1989

[8] Handbook of Mathematical Functions, edited by M. Abramowitz and I. A. Stegun. National Bureau of Standards. Applied Mathematics Series 55. 9th printing, 1970. 

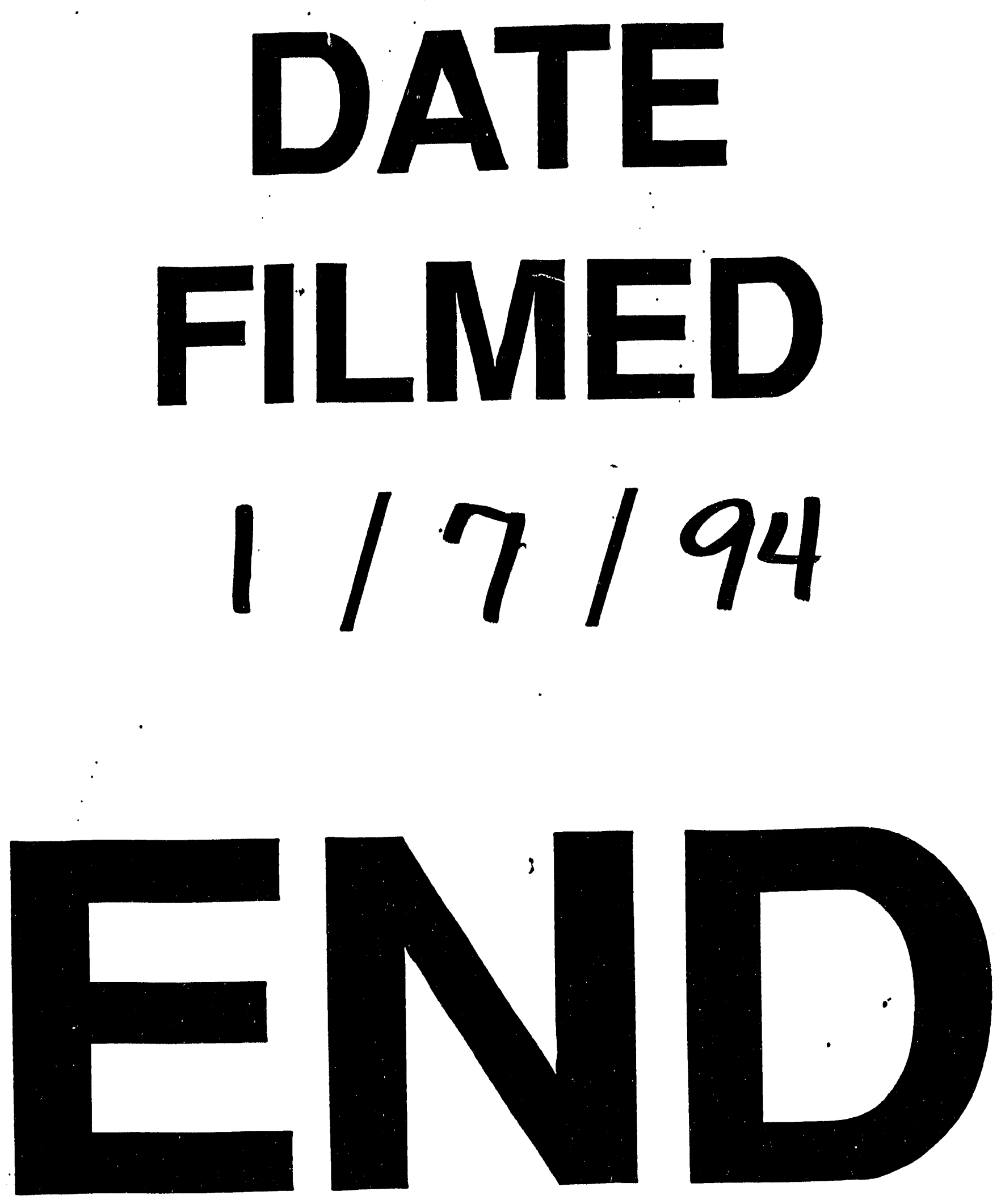
